\title{
Does exercise therapy improve pulmonary function in patients with Adolescent Idiopathic Scoliosis?
}

\section{Anthony Rafferty, Neil Fleming, Patrick Kiely, David Mockler \& Sara Dockrell}

To cite this article: Anthony Rafferty, Neil Fleming, Patrick Kiely, David Mockler \& Sara Dockrell (2022): Does exercise therapy improve pulmonary function in patients with Adolescent Idiopathic Scoliosis?, Physiotherapy Theory and Practice, DOI: 10.1080/09593985.2022.2034198

To link to this article: https://doi.org/10.1080/09593985.2022.2034198

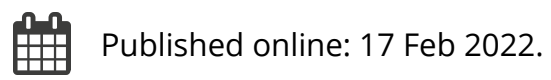

Submit your article to this journal ๘

Q View related articles $\square$

View Crossmark data ¿ 


\title{
Does exercise therapy improve pulmonary function in patients with Adolescent Idiopathic Scoliosis?
}

\author{
Anthony Rafferty MSc, $\mathrm{PT}^{\mathrm{a}}$, Neil Fleming $\mathrm{PhD}^{\mathrm{b}}$, Patrick Kiely $\mathrm{MB}, \mathrm{BCh}, \mathrm{BAO}^{c}$, David Mockler HDipLIS $\mathbb{D}^{\mathrm{d}}$, \\ and Sara Dockrell PhD, $\mathrm{PT}^{\mathrm{a}}$ \\ a'Discipline of Physiotherapy, School of Medicine, Trinity College Dublin, Trinity Centre for Health Sciences, St James's Hospital, Dublin, Ireland; \\ bepartment of Anatomy, School of Medicine, Trinity College, Level 1, Trinity Biomedical Sciences Institute, Dublin, Ireland; 'Department of \\ Orthopaedics, Children's Health Ireland at Crumlin, Dublin, Ireland; 'Trinity Research, School of Medicine, Trinity College Dublin, Trinity Centre \\ for Health Sciences, St James's Hospital, Dublin, Ireland
}

\begin{abstract}
Introduction: Exercise therapy is frequently used for treating patients with Adolescent Idiopathic Scoliosis (AIS) however no previous review has evaluated the effect of exercise therapy on pulmonary function in this population.

Objective: To systematically analyze the literature on the effect of exercise therapy on pulmonary function in patients with AIS.

Methods: A systematic electronic database search (CINAHL, Embase, Medline, Web of Science) was conducted. Manual searches of key reviews and studies were also conducted. Studies that included exercise-based interventions to improve pulmonary function in patients with AIS and reported preand post-intervention pulmonary function test scores were included. Test scores were compared using standardized mean difference (SMD) between intervention and control groups in randomized control trials $(R C T)$ and mean \pm SD between pre- and post-intervention in prospective intervention studies (PI). Methodological quality was assessed using a modified Downs and Black checklist.

Results: Fifteen studies met the inclusion criteria (six RCTs and nine PIs). Results indicated the positive effect of exercise-based therapy on lung volumes (FVC/VC) and FEV ${ }_{1}$ in patients with AIS. Conclusion: Exercise therapy has a positive effect on lung volumes in patients with AIS. The quality of many studies was only 'fair,' therefore more suitably powered higher level clinical trials are required.
\end{abstract}

\section{ARTICLE HISTORY}

Received 5 October 2021

Accepted 29 December 2021

\section{KEYWORDS}

Adolescent idiopathic

scoliosis; pulmonary

function; exercise therapy;

physiotherapeutic scoliosis-

specific exercises

\section{Introduction}

Adolescent Idiopathic Scoliosis (AIS) is a progressive deformity of the spine commencing in early puberty. It affects children from 10 years of age up to skeletal maturity and is the most common form of scoliosis, accounting for $80 \%$ of diagnosed cases (Lorente et al., 2017; Negrini et al., 2018). While prevalence data vary depending on geographic latitude (Grivas et al., 2006), the most frequently reported prevalence is $2-3 \%$ of the general population (Negrini et al., 2018) with females having a disproportionately greater risk of developing the condition (Wong, Hui, Rajan, and Chia, 2005).

Depending on the severity, AIS can result in structural deformities to the thoracic cage that impair pulmonary function (Tsiligiannis and Grivas, 2012). This impairment is most notably observed as a reduction in total lung capacity (TLC), measured either directly via dilution techniques or indirectly via forced vital capacity (FVC). In patients with moderate (Cobb Angle: 20-40 ${ }^{\circ}$ ) to severe scoliosis (Cobb Angle $>40^{\circ}$ ) a negative linear correlation has been established between the magnitude of the curve and FVC (Tsiligiannis and Grivas, 2012). However, it has also been noted that the severity of pulmonary impairments cannot be inferred from the angle of scoliosis alone (Kearon, Viviani, Kirkley, and Killian, 1993). Regardless of the cause or severity, any impaired pulmonary function is likely to negatively impact on exercise tolerance, reduce participation in physical activity and impair quality of life. Tsiligiannis and Grivas (2012) highlighted that exercise capacity is decreased even in patients with mild scoliosis and exercise dyspnea may be one of the first clinical manifestations in AIS.

The current treatment options for patients with AIS are conservative management for mild and moderate cases, and spinal fusion surgery for more severe cases. The objectives of conservative management are to halt curve progression, prevent or treat respiratory dysfunction and/or spinal pain, and correct posture (Negrini

CONTACT Anthony Rafferty raffertt@tcd.ie Discipline of Physiotherapy, School of Medicine, Trinity College Dublin, Trinity Centre for Health Sciences, St James's Hospital, Dublin D08 W9RT, Ireland

(C) 2022 Taylor \& Francis Group, LLC 
et al., 2018). Conservative management of patients with AIS typically involves a general/generic exercise program or physiotherapeutic scoliosis-specific exercise program (PSSE) and/or spinal bracing. The four PSSE programs most widely used are: 1 ) Schroth (LenhertSchroth, 1992); 2) Scientific Exercise Approach to Scoliosis (SEAS) (Romano et al., 2015); 3) Dobosiewicz (DoboMed) technique (a modification of Schroth) (Dobosiewicz, Durmala, Czernicki, and Jendrzejek, 2002); and 4) Side-shift program (Berdishevsky et al., 2016). While these programs are widely used in clinical settings, their efficacy is still widely debated.

Recent systematic reviews examining efficacy of PSSE have produced contradictory findings regarding its effect on spinal curvature (Day, Fletcher, Coghlan, and Ravine, 2019; Park, Jeon, and Park, 2018). Park, Jeon, and Park (2018) reviewed 15 studies and concluded that PSSE had a moderate effect on Cobb Angle $(d=0.65)$ and angle of trunk rotation (ATR) $(\mathrm{d}=0.53)$. However, Day, Fletcher, Coghlan, and Ravine (2019) concluded that insufficient high-quality evidence currently exists to support PSSE for improvement of Cobb angle. A more recent overview of meta-analyses concluded that the strength of evidence to support PSSE for improving either Cobb Angle or ATR was in general, weak (Zhou, Liu, Li, and Chen, 2021).

Much less is known about the efficacy of general exercise programs or PSSE on pulmonary function. No previous systematic review has focused on this topic and no meta-analysis has been performed on the cumulative pulmonary function data of all relevant studies. Park, Jeon, and Park (2018) did report a moderate effect of PSSE on vital capacity $(\mathrm{d}=0.62)$, but this effect was synthesized from only three available studies. Considering the clinical objectives of PSSE include prevention and treatment of respiratory dysfunction (Negrini et al., 2018) and the fact that many programs incorporate specific respiratory exercises (Dobosiewicz, Durmala, Czernicki, and Jendrzejek, 2002; LenhertSchroth, 1992), a more detailed understanding of the effect of exercise therapies on pulmonary function in patients with AIS is required. The purpose of this review is therefore to determine whether current evidence supports exercise-based therapy as a means of improving pulmonary function in patients with AIS.

\section{Methods}

\section{Search strategy}

A systematic search of four electronic databases (CINAHL, Embase, Medline, Web of Science) was conducted by research librarian (DM) in accordance with the Preferred Reporting Items for Systematic Reviews and Meta-Analyses (PRISMA) guidelines. The search strategy and Medical Subject Headings (MeSH) were designed for each database and comprised key search terms where "idiopathic scoliosis" was found in combination with "correction, fusion, therapy, or rehabilitation" and/or "lung, pulmonary, respiratory, or ventilation" within either the title or abstract. Search dates were restricted to January 1980-July 2020. Authors were contacted by e-mail where data were incomplete. Manual searches using bibliographies of key reviews and studies were also conducted for potentially eligible studies. The proposed review was registered at the National Institute for Health Research, PROSPERO (registration number CRD42020181193) in advance of the initial search. The final search was conducted in August 2020.

\section{Inclusion and exclusion criteria}

Studies which satisfied the following criteria were included in the systematic review. 1) Study participants had a clinical diagnosis of AIS; 2) An exercise-based intervention was performed with at least one of the following pulmonary function outcome variables measured pre- and post-intervention: Forced expiredvolume in 1 second $\left(\mathrm{FEV}_{1}\right.$ or, $\left.\mathrm{FEV}_{1} \%\right)$, Forced vital capacity (FVC or FVC\%), Vital capacity (VC or VC\%), Peak expiratory flow rate (PEF), Total lung capacity (TLC), Maximum expired flow (MEF), Forced inspired volume in 1 second $\left(\mathrm{FIV}_{1}\right)$, Forced inspiratory vital capacity (FIVC), Peak inspiratory flow rate (PIF), Forced inspiratory flow (FIF), Maximal inspiratory pressure (MIP) or maximal expiratory pressure (MEP); and 3) The study design was a randomized control trial, case-control intervention, or interventional cohort design. Case reports, editorials and literature reviews and studies that included patients with AIS who had cardiorespiratory comorbidities were excluded.

\section{Data extraction}

Studies identified by searches were imported into a reference manager (EndNote Version X9, Philadelphia, PA) and uploaded to the online platform Covidence for the title and abstract search (www.covi dence.org). Following pilot-screening to establish interrater reliability (Cohen's Kappa $=80 \%$ ), initial screening of titles and abstracts was conducted by three reviewers (TR, NF, SD). Full-text review was also carried out independently (TR and SD) with any disputes deliberated with a third reviewer (NF) until agreement was reached. Details of the RCTs (Table 1) and prospective 


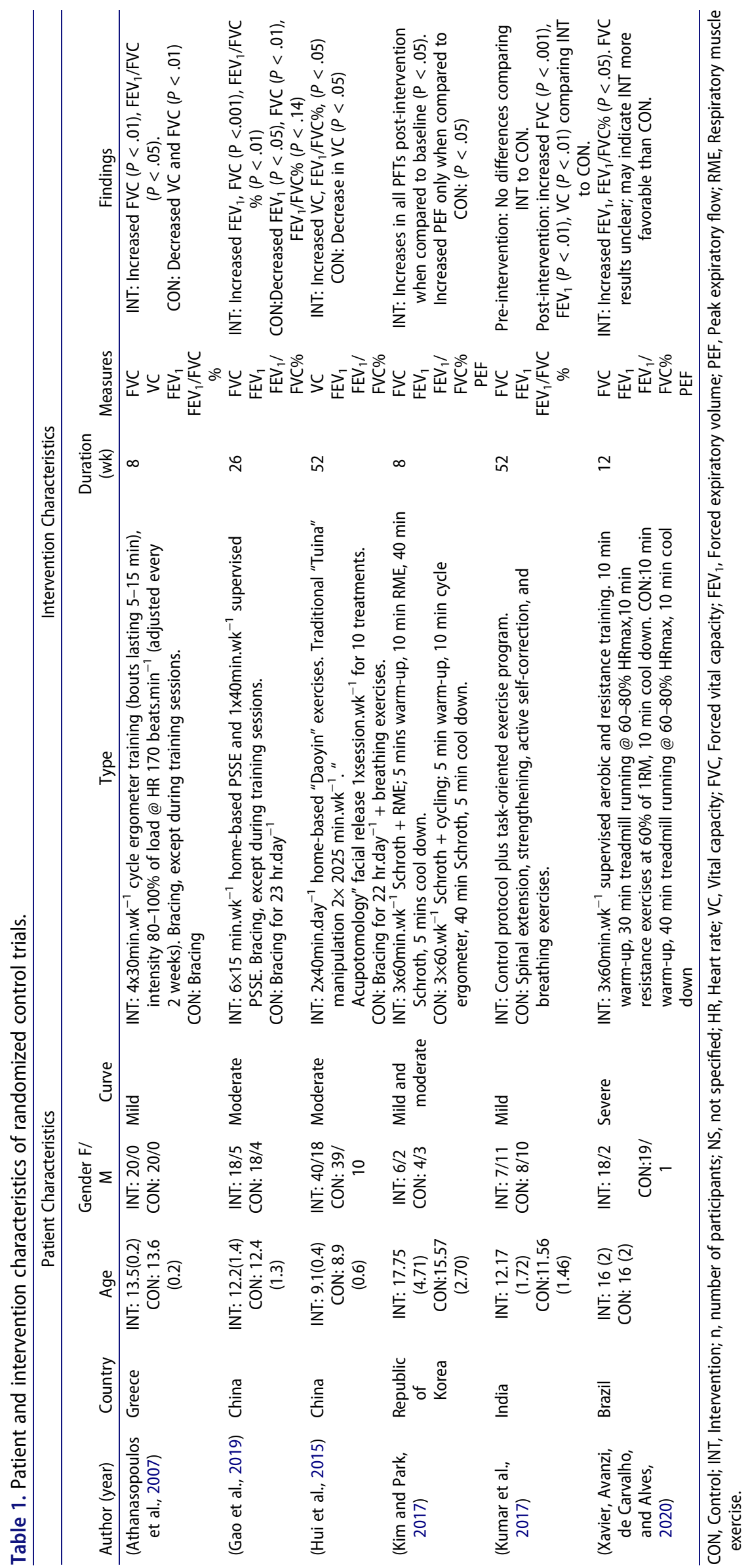


intervention (PI) studies (Table 2) were recorded in Excel and included; author, year of publication, country, patient characteristics (i.e. age, gender, and spinal curve) and intervention characteristics (i.e. type, duration, outcome measures, and findings).

\section{Quality assessment}

Each study was appraised using a modified Downs and Black Checklist that evaluates methodological strengths and weaknesses of randomized and non-randomized studies based on: 1) reporting; 2) internal validity (bias); 3) internal validity (confounding); 4) external validity; and 5) power (Downs and Black, 1998). Based on previously published descriptors (Hooper, Jutai, Strong, and Russell-Minda, 2008), studies were graded for quality as: excellent (26-28); good (20-25); fair (15$19)$; or poor $(\leq 14)$. Two independent reviewers conducted the quality assessment (TR and NF). Inter-rater disagreements were discussed until a consensus was reached.

\section{Data analysis}

Statistical analysis was conducted using Review Manager Software (RevMan Version 5.4). For the purpose of analyzing global effects, papers were allocated to one of two subgroups depending on study design. For RCTs, standardized mean difference (SMD) between pre- and post-intervention pulmonary function data was calculated. SMD and 95\% CI were subsequently compared between control and intervention groups to establish intervention effects. For PI studies, pre- and postintervention pulmonary function data were compared using random effects meta-analyses. Weighted mean difference (MD) with a $95 \%$ confidence interval $(95 \%$ CI) was calculated and intervention effect $(Z)$ estimated. The $\mathrm{I}^{2}$ statistic was used to establish the extent of heterogeneity. $\mathrm{I}^{2}$ values of $25 \%, 50 \%$, and $75 \%$ broadly representing low, moderate, and high heterogeneity (Higgins, Thompson, Deeks, and Altman, 2003). The focus of this review was on the most relevant pulmonary function tests that are carried out, namely FVC, VC and $\mathrm{FEV}_{1}$ (Graham et al., 2019) and summary findings from each meta-analysis were represented by forest plots.

\section{Results}

\section{Search results}

The initial search of four databases yielded 1,835 studies before the removal of 544 duplicates (Figure 1). Fifteen papers met the study criteria and were included in this systematic review. Six papers were identified as randomized control trials (RCT) and the remaining nine were prospective interventional (PI) studies (Tables 1 and 2, respectively). Two papers were omitted from the metaanalysis due to inappropriate presentation of data (Hui et al., 2015) and lack of reporting of standard deviation (Weiss, 1991).

Three RCTs were conducted in a hospital setting (Gao et al., 2019; Hui et al., 2015; Xavier, Avanzi, de Carvalho, and Alves, 2020) and the remaining three were in a university setting (Athanasopoulos et al., 2007; Kim and Park, 2017; Kumar et al., 2017). Five PI studies were carried out in a hospital setting (Alves, Stirbulov, and Avanzi, 2006; Amaricai et al., 2020; Fabian, 2010a, 2010b; Weiss, 1991); three in universities (Borysov and Borysov, 2012; Kim and Hwangbo, 2016; Otman, Kose, and Yakut, 2005); and one in a specialist scoliosis rehabilitation clinic (Moramarco et al., 2016). All studies measured FVC (L) or VC (L) while five RCTs and four PIs measured $\mathrm{FEV}_{1}$.

\section{Methodological quality assessment}

Output from the modified Downs and Black quality appraisal is presented in Table 3. The RCTs were classified as 'good' $(\mathrm{n}=2)$ and 'fair' $(\mathrm{n}=4)$. Five PI studies were classified as 'fair' and the remaining four studies had a rating of 'poor.' In general, studies scored well on external validity, but were of moderate quality in terms of internal validity and selection bias. In addition, only seven studies justified their sample size (Table 3).

\section{Participant characteristics}

The total sample population was 1,355 (RCT $n=283$; PI $\mathrm{n}=1072$ ). The majority of participants were female (RCT 77\%; PI 86\%). Full details of the participant characteristics are shown in Tables 1 and 2. One study did not specify participant gender (Weiss, 1991). Mean age of reported participants was $14 \pm 3$ years. One study did not provide the mean age of the participants (Weiss, 1991).

The Lenke classification for AIS spinal curves identifies six different curve types (Lenke et al., 2001). This review included two studies of participants with single thoracic curves (Athanasopoulos et al., 2007; Xavier, Avanzi, de Carvalho, and Alves, 2020) and six studies with participants who had mixed curve type including thoracic curves, lumbar curves and double curves (Alves, Stirbulov, and Avanzi, 2006; Borysov and Borysov, 2012; Fabian, 2010a, 2010b; Moramarco et al., 2016; Otman, Kose, and Yakut, 2005). The remaining seven studies did not identify the curve type of participants (Amaricai et al., 2020; Gao et al., 2019; Hui et al., 2015; Kim and Hwangbo, 2016; Kim and Park, 2017; 
Kumar et al., 2017; Weiss, 1991). Eight studies (five RCTs and three PIs) included participants with mild or moderate scoliosis (Amaricai et al., 2020; Athanasopoulos et al., 2007; Borysov and Borysov, 2012; Gao et al., 2019; Hui et al., 2015; Kim and Park, 2017; Kumar et al., 2017; Otman, Kose, and Yakut, 2005). Two studies included participants with a spectrum of AIS severity; mild, moderate and severe (Moramarco et al., 2016; Weiss, 1991). Three studies only included participants classified as severe AIS (Alves, Stirbulov, and Avanzi, 2006; Kim and Hwangbo, 2016; Xavier, Avanzi, de Carvalho, and Alves, 2020). Two studies did not specify the curve severity of the participants in their study (Fabian, 2010a, 2010b).

\section{Types of intervention}

The types of intervention used in the studies can be divided into two broad categories: 1) aerobic-based exercise programs $(\mathrm{n}=3)$ (Alves, Stirbulov, and Avanzi, 2006; Athanasopoulos et al., 2007; Xavier, Avanzi, de Carvalho, and Alves, 2020); and 2) PSSE programs designed to strengthen and straighten the spine and improve posture and breathing $(\mathrm{n}=12)$. A number of studies had additional components such as: supplementary ergonomic exercises for the intervention group (Kumar et al., 2017); Chinese traditional medicine and manual manipulation techniques (Hui et al., 2015); lower limb strengthening (Amaricai et al., 2020); or lowresistance exercises (Xavier, Avanzi, de Carvalho, and Alves, 2020) in their interventions (Tables 1 and 2).

Two studies using aerobic-based exercise programs had durations of 16 and 8 weeks, respectively (Alves, Stirbulov, and Avanzi, 2006; Athanasopoulos et al., 2007). A study using concurrent aerobic and resistance training had a duration of 12 weeks (Xavier, Avanzi, de Carvalho, and Alves, 2020). The duration of the PSSE interventions ranged from 1 week (Borysov and Borysov, 2012; Moramarco et al., 2016) to 52 weeks (Hui et al., 2015; Kumar et al., 2017; Otman, Kose, and Yakut, 2005). Two studies included a combination of supervised training and home exercises, with the programs lasting 26 and 52 weeks, respectively (Gao et al., 2019; Otman, Kose, and Yakut, 2005). The remaining studies $(\mathrm{n}=13)$ were fully supervised programs (Tables 1 and 2).

\section{Effectiveness of exercise on pulmonary function}

A fixed effects meta-analysis was conducted on five RCTs and indicated a positive effect of exercise-based interventions on lung volume (FVC and VC) in patients with AIS (SMD $=0.33,95 \%$ CI 0.46 to $0.20, \mathrm{I}^{2}=50 \%$, $P=.00001$ ) (Figure 2). The $\mathrm{I}^{2}$ score of $50 \%$ suggests moderate heterogeneity between studies. The study by
Xavier, Avanzi, de Carvalho, and Alves (2020) accounted for $42.9 \%$ of the weighting in pooled results, however they reported the lowest difference across included studies (SMD $=0.1595 \%$ CI 0.34 to -0.04 ) and results excluding this study remained significant for the intervention $(P=.00001)$. The results of a random effects meta-analysis on the PIs $(n=8)$ showed a positive effect of an exercise-based intervention (MD $=0.27,95 \%$ CI 0.37 to $\left.0.17, \mathrm{I}^{2}=10 \%, P=.00001\right)$ on FVC and VC (Figure 3). Results of a fixed-effects meta-analysis for the RCT studies in relation to $\operatorname{FEV}_{1}(n=5)$ also indicated a positive effect of exercise-based interventions $\left(\mathrm{SMD}=0.32,95 \% \mathrm{CI} 0.46\right.$ to $0.18, \mathrm{I}^{2}=32 \%$, $P=.00001$ ) (Figure 4). One of the studies could not be included as they did not report $\mathrm{FEV}_{1}$ data (Hui et al., 2015). The findings for a random effects meta-analysis of $\mathrm{FEV}_{1}$ within PIs $(\mathrm{n}=4)$ indicated a positive significant outcome favoring an exercise-based intervention $\left(\mathrm{MD}=0.19,95 \%\right.$ CI 0.34 to $\left.0.05, \mathrm{I}^{2}=0 \%, P=.00001\right)$ (Figure 5).

\section{Discussion}

The main finding of this review is that exercise-based therapy significantly improves pulmonary function in patients with AIS. Intervention duration varied greatly between studies, but improvements did not appear related to the duration of the program, with significant improvements in VC reported after as little as one week (Borysov and Borysov, 2012). In terms of quality, only two studies, both RCTs, were rated as 'good' (Gao et al., 2019; Xavier, Avanzi, de Carvalho, and Alves, 2020) with the majority of other studies found to be fair (9/15).

Testing of the pulmonary system is particularly important in individuals with AIS, given that thoracic spinal curvatures can lead to respiratory impairments (Tsiligiannis and Grivas, 2012). In patients with moderate to severe AIS, a negative linear correlation has been established between curve severity and FVC (Tsiligiannis and Grivas, 2012). However, in the absence of other underlying disorders, mild to moderate scoliosis produces very few respiratory signs or symptoms (Tsiligiannis and Grivas, 2012), despite patients with even mild AIS having impaired $\mathrm{FEV}_{1}$ and FVC compared to age-matched controls (Abdelaal et al., 2018). Individuals with impaired FVC may be entirely asymptomatic unless they are undertaking vigorous physical activity and therefore may go unnoticed without a pulmonary function test (PFT) being conducted. An undiagnosed impairment in FVC could potentially act as a barrier to participation in physical activity for patients with AIS. A recent observational study reported lower physical activity rates in patients with AIS, irrespective of curve severity, compared to 


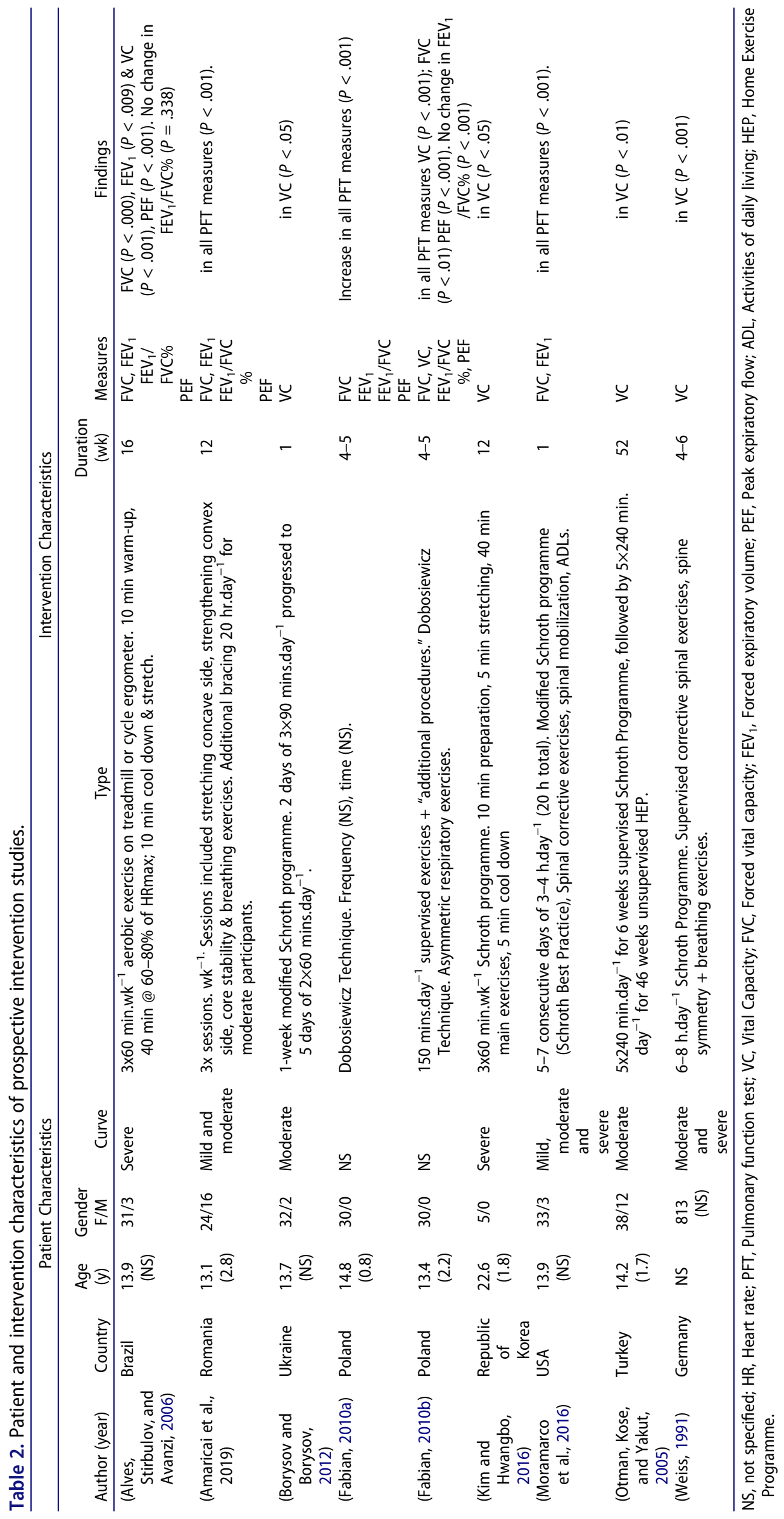




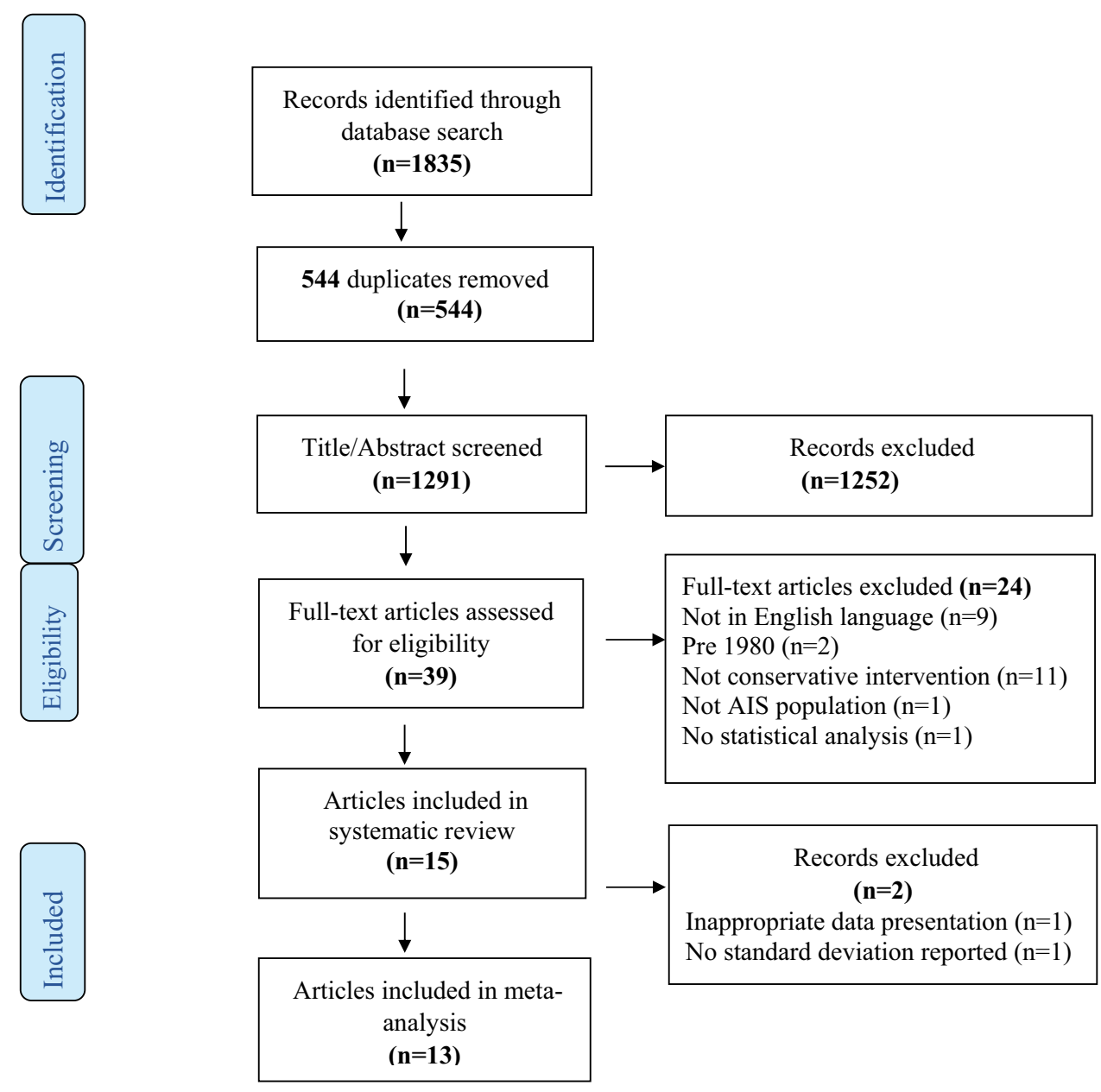

Figure 1. PRISMA flow diagram: Does exercise therapy improve pulmonary function in patients with Adolescent Idiopathic Scoliosis?.

Table 3. Risk of bias assessment using a modified downs and black checklist .

\begin{tabular}{|c|c|c|c|c|c|c|}
\hline Author (year) & Reporting (11) & External Validity (3) & Internal Validity (7) & Selection Bias (6) & Power (1) & Total (28) \\
\hline (Alves, Stirbulov, and Avanzi, 2006) & 4 & 3 & 4 & 3 & 1 & 15 \\
\hline (Amaricai et al., 2019) & 8 & 3 & 5 & 2 & 1 & 19 \\
\hline (Athanasopoulos et al., 2007) & 6 & 3 & 3 & 4 & 0 & 16 \\
\hline (Borysov and Borysov, 2012) & 6 & 3 & 2 & 3 & 1 & 15 \\
\hline (Fabian, 2010a) & 6 & 3 & 4 & 2 & 0 & 15 \\
\hline (Fabian, 2010b) & 5 & 2 & 3 & 1 & 0 & 11 \\
\hline (Gao et al., 2019) & 7 & 3 & 6 & 5 & 0 & 21 \\
\hline (Hui et al., 2015) & 7 & 3 & 3 & 4 & 1 & 18 \\
\hline (Kim and Hwangbo, 2016) & 4 & 2 & 4 & 1 & 0 & 11 \\
\hline (Kim and Park, 2017) & 5 & 3 & 4 & 3 & 0 & 15 \\
\hline (Kumar et al., 2017) & 6 & 3 & 5 & 5 & 0 & 19 \\
\hline (Moramarco et al., 2016) & 4 & 3 & 3 & 3 & 1 & 14 \\
\hline (Otman, Kose, and Yakut, 2005) & 6 & 2 & 3 & 2 & 1 & 14 \\
\hline (Weiss, 1991) & 7 & 3 & 5 & 3 & 1 & 19 \\
\hline (Xavier, Avanzi, de Carvalho, and Alves, 2020) & 10 & 3 & 7 & 4 & 0 & 24 \\
\hline
\end{tabular}

Excellent, 26-28; Good, 20-25; Fair 15-19; Poor $\leq 14$

healthy age-matched controls (Chopra et al., 2020). Considering the importance of physical activity for the health and development of adolescents (Kumar, Robinson, and Till, 2015) any impairments in pulmonary function should be quickly identified and acted upon even in patients with mild AIS. The results from this review demonstrate that completion of or participation in an exercise-based program could significantly improve FVC and $\mathrm{FEV}_{1}$ in adolescents with AIS.

The studies identified in this review were broadly categorized into two types of exercise intervention: aerobic exercise and PSSE programs. Studies using aerobic 


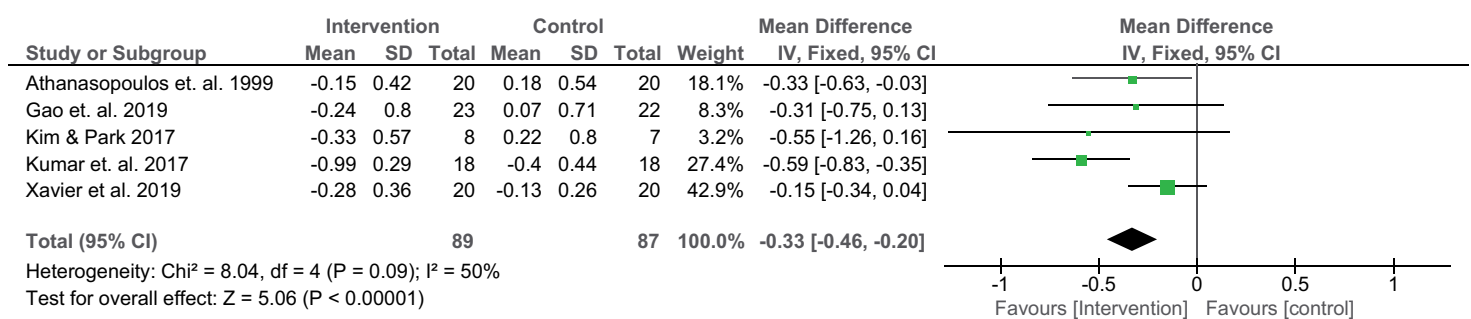

Figure 2. Forest plot depicting the cumulative effect of an exercise intervention on FVC/VC in randomized control trials (RCT).

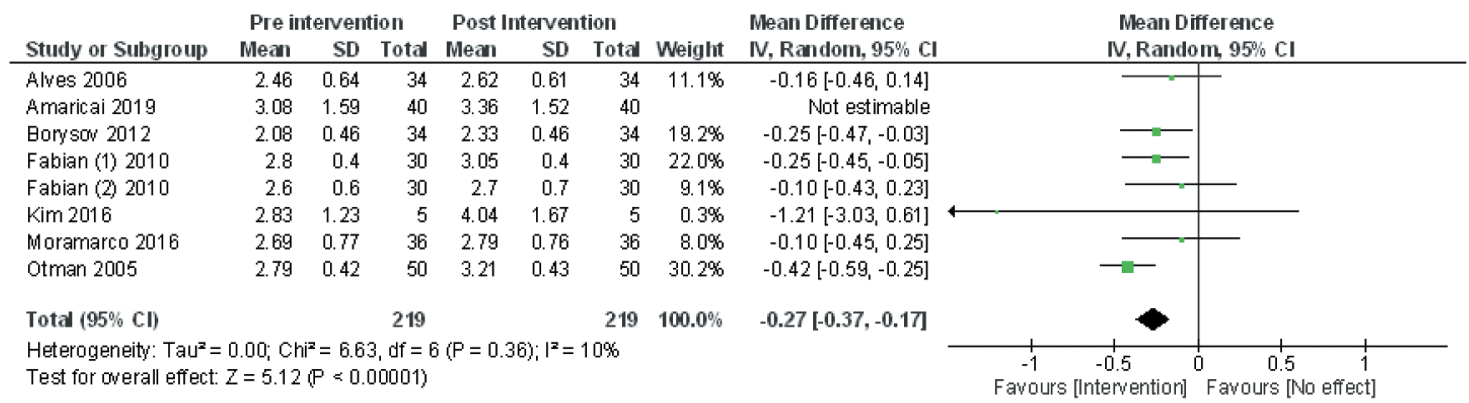

Figure 3. Forest plot depicting the cumulative effect of an exercise intervention on FVC/VC in prospective intervention studies (PI).

\begin{tabular}{|c|c|c|c|c|c|c|c|c|c|c|}
\hline \multirow[b]{2}{*}{ Study or Subgroup } & \multicolumn{3}{|c|}{ Intervention } & \multicolumn{3}{|c|}{ Control } & \multicolumn{2}{|r|}{ Mean Difference } & \multirow{2}{*}{\multicolumn{2}{|c|}{$\begin{array}{l}\text { Mean Difference } \\
\text { IV, Fixed, } 95 \% \mathrm{CI}\end{array}$}} \\
\hline & Mean & SD & Total & Mean & SD & Total & Weight & IV, Fixed, 95\% Cl & & \\
\hline Athanasopoulos et. al. 1999 & 0.02 & 0.42 & 20 & 0.09 & 0.54 & 20 & $21.7 \%$ & $-0.07[-0.37,0.23]$ & $\longrightarrow$ & \\
\hline Gao et. al. 2019 & -0.26 & 0.75 & 23 & 0.06 & 0.66 & 20 & $11.0 \%$ & $-0.32[-0.74,0.10]$ & & - \\
\hline Kim \& Park 2017 & -0.39 & 0.5 & 8 & 0.05 & 0.94 & 7 & $3.2 \%$ & $-0.44[-1.22,0.34]$ & & \\
\hline Kumar et. al. 2017 & -0.78 & 0.36 & 18 & -0.23 & 0.44 & 18 & $28.3 \%$ & $-0.55[-0.81,-0.29]$ & & \\
\hline Xavier et al. 2019 & -0.38 & 0.47 & 20 & -0.11 & 0.25 & 20 & $35.8 \%$ & $-0.27[-0.50,-0.04]$ & & \\
\hline Total $(95 \% \mathrm{Cl})$ & & & 89 & & & 85 & $100.0 \%$ & $-0.32[-0.46,-0.18]$ & & \\
\hline $\begin{array}{l}\text { Heterogeneity: } \mathrm{Chi}^{2}=5.88, \mathrm{~d} \\
\text { Test for overall effect: } Z=4.4\end{array}$ & $\begin{array}{l}=4(P= \\
(P<0 .\end{array}$ & $\begin{array}{l}0.21) \\
00001)\end{array}$ & $1^{2}=32$ & & & & & & $\begin{array}{ccc}-1 & -0.5 & 0 \\
\text { Favours [Intervention] }\end{array}$ & $\begin{array}{c}0.5 \\
\text { Favours [control] }\end{array}$ \\
\hline
\end{tabular}

Figure 4. Forest plot depicting the cumulative effect of an exercise intervention on $\mathrm{FEV}_{1}$ in randomized control trials (RCT).

\begin{tabular}{|c|c|c|c|c|c|c|c|c|c|c|c|}
\hline \multirow[b]{2}{*}{ Study or Subgroup } & \multicolumn{3}{|c|}{ Pre Intervention } & \multicolumn{3}{|c|}{ Post Intervention } & \multirow[b]{2}{*}{ Weight } & \multirow{2}{*}{$\begin{array}{l}\text { Mean Difference } \\
\text { IV, Random, } 95 \% \mathrm{CI}\end{array}$} & \multirow{2}{*}{\multicolumn{3}{|c|}{$\begin{array}{l}\text { Mean Difference } \\
\text { IV, Rando }, 95 \% \mathrm{CI}\end{array}$}} \\
\hline & Mean & SD & Total & Mean & SD & Total & & & & & \\
\hline Alves 2006 & 2.13 & 0.55 & 34 & 2.27 & 0.51 & 34 & $32.7 \%$ & $-0.14[-0.39,0.11]$ & & & \\
\hline Amaricai 2019 & 2.82 & 1.17 & 40 & 2.94 & 1.27 & 40 & $7.3 \%$ & $-0.12[-0.66,0.42]$ & & & \\
\hline Borysow 2012 & 0 & 0 & 0 & 0 & 0 & 0 & & Not estimable & & & \\
\hline Fabian (1) 2010 & 2.1 & 0.6 & 30 & 2.5 & 0.5 & 30 & $26.6 \%$ & $-0.40[-0.68,-0.12]$ & & & \\
\hline Fabian (2) 2010 & 0 & 0 & 0 & 0 & 0 & 0 & & Not estimable & & & \\
\hline Kim 2016 & 0 & 0 & 0 & 0 & 0 & 0 & & Not estimable & & & \\
\hline Moramarco 2016 & 2.28 & 0.55 & 36 & 2.37 & 0.53 & 36 & $33.4 \%$ & $-0.09[-0.34,0.16]$ & & & \\
\hline Otman 2005 & 0 & 0 & 0 & 0 & 0 & 0 & & Not estimable & & & \\
\hline Total $(95 \% \mathrm{Cl})$ & & & 140 & & & 140 & $100.0 \%$ & $-0.19[-0.34,-0.05]$ & & & \\
\hline \multicolumn{9}{|c|}{$\begin{array}{l}\text { Heterogeneity: Tau }{ }^{2}=0.00 ; \mathrm{Chi}^{2}=3.00, \mathrm{df}=3(\mathrm{P}=0.39) ; \mathrm{I}^{2}=0 \% \\
\text { Test for owerall effect: } Z=2.60(P=0.009)\end{array}$} & $\begin{array}{cc}1 & -0.5 \\
\text { Favours [Intervention] }\end{array}$ & $\begin{array}{ll} & 1 \\
\text { Favours [No effect] }\end{array}$ & 1 \\
\hline
\end{tabular}

Figure 5. Forest plot depicting the cumulative effect of an exercise intervention on $\mathrm{FEV}_{1}$ in prospective intervention studies (PI).

exercise reported significant improvements in FVC/VC (Alves, Stirbulov, and Avanzi, 2006; Athanasopoulos et al., 2007; Xavier, Avanzi, de Carvalho, and Alves, 2020). Aerobic fitness has been shown to be positively correlated with improvements in FVC, VC and $\mathrm{FEV}_{1}$ in children and adolescents regardless of height, weight or gender (Hancox and Rasmussen, 2018). Previous studies have shown improvements in $\mathrm{FEV}_{1}$ from high-intensity exercise programs (Rawashdeh and Alnawaiseh, 2018).
Physiotherapeutic scoliosis-specific exercise programs are frequently used in the management of spinal curve progression in scoliosis (Berdishevsky et al., 2016; Negrini et al., 2018). Despite the methodological variability, all 12 studies in this review that included a PSSE intervention reported significant improvements in FVC or VC. A number of studies postulated that improvement in pulmonary function following a PSSE program was related to addressing the abnormal breathing 
patterns that occur in patients with scoliosis because of reduced chest expansion and restricted rib mobility (Borysov and Borysov, 2012; Moramarco et al., 2016). Weiss (1991) also proposed the concept of abnormal chest cage mechanics leading to dysfunctional breathing. Interestingly, Xavier, Avanzi, de Carvalho, and Alves (2020) who included resistance exercises in their intervention group found that participants with severe AIS had better outcomes when aerobic exercises were combined with anaerobic workouts. Similar findings have also been found in other non-AIS-based cohorts (Khosravi, Tayebi, and Safari, 2013).

The intervention duration does not seem to be a factor in improvements in post-intervention PFTs. The most significant improvements in FVC/VC were reported in an RCT with an 8-week intervention (Alves, Stirbulov, and Avanzi, 2006) and one with a 52-week intervention (Athanasopoulos et al., 2007). Similarly, PIs with the most significant results were studies with a 4-week intervention (Fabian, 2010b), a 4-6 week intervention (Weiss, 1991), and a 52-week intervention (Otman, Kose, and Yakut, 2005). Results from studies comparing absolute pulmonary data, over longer timeperiods would need to be viewed with caution. Individual's lung volumes progressively increase from birth until pulmonary maturity is reached at approximately 20 years of age (Barroso, Martín, Romero, and Ruiz, 2018). The pattern of development is linear with age and height until adolescence is reached, at approximately 10 years for females and 12 years for males (Neve, Girard, Flahault, and Boulé, 2002). In adolescence, a number of other factors have been shown to influence lung capacity such as, height, weight, gender and racial background (Barroso, Martín, Romero, and Ruiz, 2018; Neve, Girard, Flahault, and Boulé, 2002). A direct comparison between absolute pulmonary function tests before and after an intervention takes no account of the factors affecting lung growth and may overstate the respiratory improvements of an intervention (Neve, Girard, Flahault, and Boulé, 2002). The potential for error when using absolute data increases as the duration of the intervention increases. Since stature and arm span are independent predictors of pulmonary function (Golshan et al., 2007), it is suggested normalizing PFT data to arm span might provide a more accurate estimate of any changes brought about by longer-term interventions (Graham et al., 2019)

\section{Limitations}

A number of factors limit this review. Firstly, studies included a combination of RCTs and PIs with participants who had varying curve severity ranging from mild to severe AIS. Caution is therefore required when interpreting conclusions presented. Secondly, only four studies reported results in relative values. Relative or predicted pulmonary values are recommended for use in pulmonary analysis and take into account child and adolescent development over time. In particular, it is recommended that $\mathrm{FEV}_{1}$ is reported as a percentage of predicted or as a z-score when performing test-retest comparisons, to avoid gender or height bias (Graham et al., 2019). Finally, the review focused on primary pulmonary function test results, $\mathrm{FVC}, \mathrm{VC}$ and $\mathrm{FEV}_{1}$ as key variables that highlight pulmonary capacity in individuals with scoliosis, although several studies carried out a number of other PFTs not quantitatively evaluated in this review.

\section{Conclusion}

This is the first systematic review and meta-analysis directly evaluating the effect of exercise-based treatment on pulmonary function in patients with AIS. The results of our meta-analysis indicated a positive effect of exercisebased interventions (i.e. aerobic and PSSE) on lung volumes $(\mathrm{FVC} / \mathrm{VC})$ and $\mathrm{FEV}_{1}$ in patients diagnosed with AIS. The quality of the majority of studies in this review was only 'fair,' therefore more suitably powered higher level clinical trials, using normalized pulmonary function measures and clearly defined groupings of curve severity are required.

\section{Disclosure statement}

No potential conflict of interest was reported by the author(s).

\section{Funding}

The author(s) reported there is no funding associated with the work featured in this article.

\section{ORCID}

David Mockler HDipLIS (D) http://orcid.org/0000-0001-61718751 


\section{References}

Abdelaal A, Kafy E, Elayat M, Sabbahi M, Badghish M 2018 Changes in pulmonary function and functional capacity in adolescents with mild idiopathic scoliosis: Observational cohort study. Journal of International Medical Research 46: 381-391. doi:10.1177/0300060517715375.

Alves VLDS, Stirbulov R, Avanzi O 2006 Impact of a physical rehabilitation program on the respiratory function of adolescents with idiopathic scoliosis. Chest 130(2): 500-505. doi:10.1016/S0012-3692(15)51867-9.

Amaricai E, Suciu O, Onofrei R, Miclaus R, Iacob R, Cațan L, Popoiu C, Cerbu S, Boia E 2020 Respiratory function, functional capacity, and physical activity behaviours in children and adolescents with scoliosis. Journal of International Medical Research 48: 300060519895093. doi:10.1177/0300060519895093.

Athanasopoulos S, Paxinos T, Tsafantakis E, Zachariou K, Chatziconstantinou S 2007 The effect of aerobic training in girls with idiopathic scoliosis. Scandinavian Journal of Medicine \& Science in Sports 9(1): 36-40. doi:10.1111/ j.1600-0838.1999.tb00204.x.

Barroso A, Martín E, Romero L, Ruiz F 2018 Factors affecting lung function: A review of the literature. Archivos de Bronconeumología 54: 327-332. doi:10.1016/j. arbr.2018.04.003.

Berdishevsky H, Lebel V, Bettany-Saltikov J, Rigo M, Lebel A, Hennes A, Romano M, Białek M, M'hango A, Betts T, et al. 2016 Physiotherapy scoliosis-specific exercises A comprehensive review of seven major schools. Scoliosis and Spinal Disorders 11(1): 20. doi:10.1186/s13013-0160076-9.

Borysov M, Borysov A 2012 Scoliosis short-term rehabilitation (SSTR) according to 'Best Practice' standards-are the results repeatable? Scoliosis 7: 1. doi:10.1186/1748-7161-7-1.

Chopra S, Larson A, Kaufman K, Milbrandt T, Bergman P 2020 Accelerometer based assessment of daily physical activity and sedentary time in adolescents with idiopathic scoliosis. PLoS One 15(9): e0238181. doi:10.1371/journal. pone.0238181.

Day J, Fletcher J, Coghlan M, Ravine T 2019 Review of scoliosis-specific exercise methods used to correct adolescent idiopathic scoliosis. Archives of Physiotherapy 9: 8. doi:10.1186/s40945-019-0060-9.

Dobosiewicz K, Durmala J, Czernicki K, Jendrzejek H 2002 Pathomechanic basics of conservative treatment of progressive idiopathic scoliosis according to Dobosiewicz method based upon radiologic evaluation. Studies in Health Technology and Informatics 91: 336-341.

Downs S, Black N 1998 The feasibility of creating a checklist for the assessment of the methodological quality both of randomised and non-randomised studies of health care interventions. Journal of Epidemiology and Community Health 52: 377-384. doi:10.1136/jech.52.6.377.

Fabian K 2010a Evaluation of lung function, chest mobility, and physical fitness during rehabilitation of scoliotic girls. Ortopedia, Traumatologia, Rehabilitacja 12: 301-309.

Fabian K 2010b Evaluation of the effectiveness of asymmetric breathing exercises according to Dobosiewicz on chosen functional parameters of the respiratory system in girls with scoliosis. Physiotherapy 18(4): 212-216. doi:10.2478/ v10109-010-0072-1.
Gao C, Zheng Y, Fan C, Yang Y, He C, Wong M 2019 Could the clinical effectiveness be improved under the integration of orthotic intervention and scoliosis-specific exercise in managing adolescent idiopathic scoliosis? A randomized controlled trial study. American Journal of Physical Medicine \& Rehabilitation 98: 642-648. doi:10.1097/ PHM.0000000000001160.

Golshan M, Crapo R, Amra B, Jensen R, Golshan R 2007 Arm span as an independent predictor of pulmonary function parameters: Validation and reference values. Respirology 12(3): 361-366. doi:10.1111/j.14401843.2007.01070.x.

Graham B, Steenbruggen I, Miller M, Barjaktarevic I, Cooper B, Hall G, Hallstrand T, Kaminsky D, McCarthy K, McCormack M, et al. 2019 Standardization of spirometry 2019 update. An Official American Thoracic Society and European Respiratory Society Technical Statement. American Journal of Respiratory and Critical Care Medicine 200: 70-88. doi:10.1164/rccm.201908-1590ST.

Grivas T, Vasiliadis E, Mouzakis V, Mihas C, Koufopoulos G 2006 Affiliations expand association between adolescent idiopathic scoliosis prevalence and age at menarche in different geographic latitudes. Scoliosis 1(1): 9. doi:10.1186/1748-7161-1-9.

Hancox R, Rasmussen F 2018 Does physical fitness enhance lung function in children and young adults? European Respiratory Journal 51: 1701374. doi:10.1183/ 13993003.01374-2017.

Higgins J, Thompson S, Deeks J, Altman D 2003 Measuring inconsistency in meta-analyses. Bmj 327(7414): 557-560. doi:10.1136/bmj.327.7414.557.

Hooper P, Jutai J, Strong G, Russell-Minda E 2008 Age-related macular degeneration and low-vision rehabilitation: A systematic review. Canadian Journal of Ophthalmology 43: 180-187. doi:10.3129/i08-001.

Hui W, Jinyuan X, Zhong J, Shuliang Y, Hongquan S, Xitao N, Huanmin H, Wei C, Jianwei P, Nengyi J, et al. 2015 Effect of a Traditional Chinese Medicine combined therapy on adolescent idiopathic scoliosis: A randomized controlled trial. Journal of Traditional Chinese Medicine 35(5): 514-519. doi:10.1016/S0254-6272(15)30133-3.

Kearon C, Viviani G, Kirkley A, Killian K 1993 Factors determining pulmonary function in adolescent idiopathic thoracic scoliosis. American Review of Respiratory Disease 148: 288-294. doi:10.1164/ajrccm/148.2.288.

Khosravi M, Tayebi S, Safari H 2013 Single and concurrent effects of endurance and resistance training on pulmonary function. Iranian Journal of Basic Medical Sciences 16(4): 628-634.

Kim K, Hwangbo P 2016 Effects of the Schroth exercise on the Cobb's angle and vital capacity of patients with idiopathic scoliosis that is an operative indication. Journal of Physical Therapy Science 28: 923-926. doi:10.1589/jpts.28.923.

Kim M-J, Park D-S 2017 The effect of Schroth's threedimensional exercises in combination with respiratory muscle exercise on Cobb's angle and pulmonary function in patients with idiopathic scoliosis. Physical Therapy Rehabilitation Science 6(3): 113-119. doi:10.14474/ ptrs.2017.6.3.113.

Kumar A, Kumar S, Sharma V, Srivastava R, Gupta A, Parihar A, Verma V, Kumar D 2017 Efficacy of taskoriented exercise program based on ergonomics on Cobb's Angle and pulmonary function improvement in adolescent 
idiopathic scoliosis - A randomized control trial. Journal of Clinical and Diagnostic Research 11: YC01-YC04. doi:10.7860/JCDR/2017/27497.10335.

Kumar B, Robinson R, Till S 2015 Physical activity and health in adolescence. Clinical Medicine 15(3): 267-272. doi:10.7861/clinmedicine.15-3-267.

Lenhert-Schroth C 1992 Introduction to the three-dimensional scoliosis treatment according to Schroth. Physiotherapy 78: 810-815. doi:10.1016/S00319406(10)60451-8.

Lenke L, Betz R, Harms J, Bridwell K, Clements D, Lowe T, Blanke K 2001 Adolescent idiopathic scoliosis. A new classification to determine extent of spinal arthrodesis. Journal of Bone and Joint Surgery 83(8): 1169-1181. doi:10.2106/ 00004623-200108000-00006.

Lorente A, Barrios C, Burgos J, Hevia E, FernándezPineda L, Lorente R, Rosa B, Pérez-Encinas C 2017 Cardiorespiratory function does not improve 2 years after posterior surgical correction of adolescent idiopathic scoliosis. Spine 42: 1391-1397. doi:10.1097/ BRS.0000000000002105.

Moramarco M, Fadzan M, Moramarco K, Heller A, Righter S 2016 The influence of short-term scoliosis-specific exercise rehabilitation on pulmonary function in patients with AIS. $<$ ![CDATA[Current Pediatric Reviews]]> 12(1): 17-23. doi:10.2174/1573396312666151117120514.

Negrini S, Donzelli S, Aulisa A, Czaprowski D, Schreiber S, de Mauroy J, Diers H, Grivas T, Knott P, Kotwicki T, et al. 20182016 SOSORT guidelines: Orthopaedic and rehabilitation treatment of idiopathic scoliosis during growth. Scoliosis and Spinal Disorders 13: 3. doi:10.1186/s13013017-0145-8.

Neve V, Girard F, Flahault A, Boulé M 2002 Lung and thorax development during adolescence: Relationship with pubertal status. European Respiratory Journal 20(5): 1292-1298. doi:10.1183/09031936.02.00208102.
Otman S, Kose N, Yakut Y 2005 The efficacy of Schroth's 3-dimensional exercise therapy in the treatment of adolescent idiopathic scoliosis in Turkey. Saudi Medical Journal 26: 1429-1435.

Park J, Jeon H, Park H 2018 Effects of the Schroth exercise on idiopathic scoliosis: A meta-analysis. European Journal of Physical and Rehabilitation Medicine 54(3): 440-449. doi:10.23736/S1973-9087.17.04461-6.

Rawashdeh A, Alnawaiseh N 2018 The effect of high-intensity aerobic exercise on the pulmonary function among inactive male individuals. Biomedical and Pharmacology Journal 11: 735-741. doi:10.13005/bpj/1427.

Romano M, Negrini A, Parzini S, Tavernaro M, Zaina F, Donzelli S, Negrini S 2015 SEAS (Scientific Exercises Approach to Scoliosis): A modern and effective evidence based approach to physiotherapic specific scoliosis exercises. Scoliosis 10(1): 3. doi:10.1186/s13013-014-0027-2.

Tsiligiannis T, Grivas T 2012 Pulmonary function in children with idiopathic scoliosis. Scoliosis 7: 7. doi:10.1186/17487161-7-7.

Weiss H 1991 The Effect of an exercise program on vital capacity and rib mobility in patients with idiopathic scoliosis. Spine 16 (1): 88-93. doi:10.1097/00007632-199101000-00016.

Wong HK, Hui J, Rajan U, Chia HP 2005 Idiopathic scoliosis in Singapore schoolchildren. A prevalence study 15 years into the screening program. Spine 30: 1188-1196. doi:10.1097/01.brs.0000162280.95076.bb.

Xavier V, Avanzi O, de Carvalho B, Alves V 2020 Combined aerobic and resistance training improves respiratory and exercise outcomes more than aerobic training in adolescents with idiopathic scoliosis: A randomised trial. Journal of Physiotherapy 66(1): 33-38. doi:10.1016/j.jphys.2019.11.012.

Zhou Z, Liu F, Li R, Chen X 2021 The effects of exercise therapy on adolescent idiopathic scoliosis: An overview of systematic reviews and meta-analyses. Complementary Therapies in Medicine 58: 102697. doi:10.1016/j. ctim.2021.102697. 